\author{
Boleseaw Ciesielski (Boston) \\ ZBigniew Ciesielski (Sopot)
}

\title{
IMAGE COMPRESSION WITH SCHAUDER BASES
}

Abstract. As is known, color images are represented as multiple channels, i.e. integer-valued functions on a discrete rectangle corresponding to pixels on the screen. Thus, image compression can be reduced to investigating suitable properties of such functions. Each channel is compressed independently. We are representing each such function by means of multi-dimensional Haar and diamond bases so that the functions can be remembered by their basis coefficients without loss of information. For each of the two bases we present in detail the algorithms for calculating the basis coefficients and conversely, for recovering the functions from the coefficients. Next, we use the fact that both the bases are greedy in suitable Besov norms and apply thresholding to compress the information carried by the coefficients. After this operation on the basis coefficients the corresponding approximation of the image can be obtained. The principles of these algorithms are known (see e.g. [3]

) but the details seem to be new. Moreover, our philosophy of applying approximation theory is different. The principal assumption is that the input data come from some images. Approximation theory, mainly the isomorphisms between Besov function spaces and suitable sequence spaces given by the Haar and diamond bases (see [1]

, [2]

), and the greediness of these bases, are used only to choose a proper norm in the space of images. The norm is always finite and it is used for thresholding only.

1. Introduction. In this paper we investigate functions over a discrete $d$-dimensional cube $Q^{d}$ where $Q$ is an interval of integers. The dimension of

2000 Mathematics Subject Classification: 26A16, 26B35, 41A15, 49M25, 68U10, $94 \mathrm{~A} 08$. mation.

Key words and phrases: Hölder, compression, spline approximation, discrete approxi- 
the vector space of all real-valued functions over $Q^{d}$ is $|Q|^{d}$ where $|Q|$ is the cardinality of $Q$. In this function space we construct the Haar basis and the diamond basis. The first one is orthogonal in the natural scalar product, and the second one is interpolating at the points of the domain $Q^{d}$. For each of the bases we describe two algorithms: The first one computes the coefficients of the basis expansion of a given function. The second one computes the values of a function given its expansion, i.e. from its basis coefficients. In the last part, motivated by theoretical results presented in Section 2, we consider suitable greedy norms for both the bases in question. This, after introducing thresholding, permits us to introduce a criterion for replacing small basis coefficients by zeros, and then using the second algorithm to produce a function which is an approximant of the original one and corresponds to the given value of the thresholding. We mention that the algorithms in the diamond case are implemented for the compression of images.

2. Theoretical background. We start by recalling some notions relating to Schauder bases in Banach spaces (see e.g. [8]). An abstract Banach space $X$ with the norm $\|\cdot\|_{X}$ is denoted as $\left[X,\|\cdot\|_{X}\right]$. A sequence $\left\{x_{n}\right\}=$ $\left\{x_{n}: n=0,1, \ldots\right\}$ in $\left[X,\|\cdot\|_{X}\right]$ is called a Schauder basis, or simply a basis, in $X$ if for each $x \in X$ there is a unique sequence $\underline{a}=\left\{a_{n}\right\}=\left\{a_{n}, n=0,1, \ldots\right\}$ of scalars such that

$$
x=\sum_{n=0}^{\infty} a_{n} x_{n} .
$$

For a given basis $\left\{x_{n}\right\}$ there are unique linear functionals $\left\{x_{n}^{*}\right\}$ in the dual space $X^{*}$ such that $a_{n}=x_{n}^{*}(x)$. The system $\left\{x_{0}, x_{1}, \ldots ; x_{0}^{*}, x_{1}^{*}, \ldots\right\}$ is biorthonormal, i.e. $x_{k}^{*}\left(x_{i}\right)=\delta_{k, i}$. Now, denote by $\mathcal{A}$ the set of all $\underline{a}$ appearing in (2.1) while $x$ runs through $X$. The linear space $\mathcal{A}$ with the norm

$$
\|\underline{a}\|_{\mathcal{A}}=\sup _{n \geq 0}\left\|\sum_{i=0}^{n} a_{i} x_{i}\right\|_{X}
$$

is a Banach space linearly isomorphic to $X$. The Banach space $\left[\mathcal{A},\|\cdot\|_{\mathcal{A}}\right]$ is customarily called the coefficient space. The basis $\left\{x_{n}\right\}$ is called unconditional if for each $x \in X$ the series on the right hand side of (2.1) converges unconditionally, that is,

$$
\sum_{n=0}^{\infty} \varepsilon_{n} a_{n} x_{n}
$$

converges for each $\underline{\varepsilon}=\left\{\varepsilon_{n}: n=0,1, \ldots\right\}$ with $\varepsilon_{n}= \pm$. There are two more recent definitions of special bases in Banach spaces which are important for approximations and which we recall here. A normalized basis $\left\{x_{n}\right\}$ in the Banach space $X$, i.e. such that $\left\|x_{n}\right\|_{X}=1$, is said to be democratic if there is 
a constant $C>0$ such that for any two subsets $P$ and $Q$ of natural numbers of finite equal cardinality we have

$$
\frac{1}{C}\left\|\sum_{n \in P} x_{n}\right\|_{X} \leq\left\|\sum_{n \in Q} x_{n}\right\|_{X} \leq C\left\|\sum_{n \in P} x_{n}\right\|_{X} .
$$

For the second definition we introduce for given $x \in X$ and for given natural $m$ the best nonlinear approximation of order $m$, i.e.

$$
\mathcal{E}_{m}(x, X)=\inf _{\# G=m} \inf _{c_{n} \in \mathbb{R}}\left\|x-\sum_{n \in G} c_{n} x_{n}\right\|_{X},
$$

where $G$ denotes a subset of the natural numbers. Now, a normalized basis $\left\{x_{n}\right\}$ in $X$ is called greedy if there is a finite constant $C$ such that

$$
\mathcal{E}_{m}(x, X) \leq C\left\|x-\sum_{n \in G_{m}(x)} a_{n} x_{n}\right\|_{X}
$$

for each $x \in X$ and each set $G_{m}(x)$ of indices of cardinality $m$ such that

$$
\min \left\{\left|a_{n}\right|: n \in G_{m}(x)\right\} \geq \max \left\{\left|a_{n}\right|: n \notin G_{m}(x)\right\} .
$$

We state the nice result of S. V. Konyagin and V. N. Temlyakov on greedy bases [4]:

THEOREM 2.1. A normalized basis in a Banach space is greedy if and only if it is democratic and unconditional.

We now confine our attention to a special class of function spaces on the $d$-dimensional cube $I^{d}=[0,1]^{d}$, namely to Besov spaces. There are various definitions of these spaces, usually they depend on three parameters: $\alpha$ - the smoothness parameter, $p$ - the integrability exponent, and $q$ - the averaging exponent. In order to write down the definition of the Besov norm we need a modulus of smoothness. For a function $f: I^{d} \rightarrow \mathbb{R}$ and a vector $h \in \mathbb{R}^{d}$, we denote by $\Delta_{h} f$ the progressive difference of $f$ with increment $h$ :

$$
\Delta_{h} f(t)= \begin{cases}f(t+h)-f(t) & \text { if } t, t+h \in I^{d} \\ 0 & \text { otherwise. }\end{cases}
$$

If $f \in L^{p}\left(I^{d}\right), 1 \leq p \leq \infty$, and $t>0$, then the $L^{p}$ modulus of smoothness is defined as

$$
\omega_{p}(f ; t)=\sup _{0<|h|<t}\left(\int_{I^{d}}\left|\Delta_{h} f(x)\right|^{p} d x\right)^{1 / p},
$$

where $|h|$ is the Euclidean length of $h \in \mathbb{R}^{d}$. Now, for $f \in L^{p}\left(I^{d}\right)$, the Besov norm is defined by

$$
\|f\|_{p, q}^{(\alpha)}=\left(\int_{0}^{1}\left(\frac{\omega_{p}(f ; t)}{t^{\alpha}}\right)^{q} \frac{d t}{t}\right)^{1 / q}
$$


The Banach space of $f \in L^{p}\left(I^{d}\right)$ with (2.10) finite is called the Besov space and is denoted by $B_{p, q}^{\alpha}=B_{p, q}^{\alpha}\left(I^{d}\right)$. Note that $B_{p, q}^{\alpha}$ is a separable Banach space for each triple of parameters $0<\alpha \leq 1,1 \leq p, q<\infty$, and this is the range to which we restrict our attention below.

To each of the two algorithms for image compression there corresponds a basis in $B_{p, q}^{\alpha}$. We start with the description of the diamond basis and then we pass to the Haar basis. To define the diamond basis over $I^{d}$ we start with the function $\psi(t)=\max [0,1-|t|]$ and with the set $D$ of all dyadic points in $I$. Define $D_{0}=\{0,1\}$ and $D_{k}=\left\{(2 j-1) / 2^{k}: j=1, \ldots, 2^{k-1}\right\}$ for $k=1,2, \ldots$ Thus

$$
D=\bigcup_{k \geq 0} D_{k}
$$

and the Faber-Schauder functions over $I$ are defined as follows:

$$
\phi_{\tau}(t)=\psi\left(2^{k}(t-\tau)\right) \quad \text { for } \tau \in D_{k}, k=0,1, \ldots
$$

For the diamond functions over $I^{d}$ it is convenient to set $C_{0}=D_{0}$ and $C_{k}=C_{k-1} \cup D_{k}$. Then

$$
C_{k}^{d}=C_{k-1}^{d} \cup D_{k, d}
$$

where

$$
D_{k, d}=\left\{\underline{\tau}=\left(\tau_{1}, \ldots, \tau_{d}\right) \in C_{k}^{d}: \exists_{i} \tau_{i} \in D_{k}\right\}, \quad D_{0, d}=D_{0}^{d} .
$$

Now, define

$$
\phi_{\underline{\tau}}(\underline{t})=\prod_{i=1}^{d} \psi\left(2^{k}\left(t_{i}-\tau_{i}\right)\right) \quad \text { for } \underline{\tau} \in D_{k, d}, k=0,1, \ldots
$$

In the two-dimensional case all the basis functions restricted to $I^{2}$ can be easily graphically represented, and they look like diamonds. The system $\left\{\phi_{\bar{\tau}}: \bar{\tau} \in D^{d}\right\}$ is called the diamond or multi-affine basis, and when ordered in a sequence in such a way that $D_{k, d}$ precedes $D_{k+1, d}$, it is a basis in the Banach space $C\left(I^{d}\right)$ of continuous functions over $I^{d}$ (cf. [5], [7]). We mention some of its properties. For each $f \in C\left(I^{d}\right)$ there is a unique collection $\left\{b_{\underline{\tau}}: \underline{\tau} \in D_{k, d}, k=0,1, \ldots\right\}$ of real numbers such that

$$
f=\sum_{k=0}^{\infty} \sum_{\underline{\tau} \in D_{k, d}} b_{\underline{\tau}} \phi_{\underline{\underline{\tau}}}
$$

and the series converges uniformly over the cube $I^{d}$ (i.e. in the norm of $\left.C\left(I^{d}\right)\right)$. The coefficients $b_{\underline{\tau}}=b_{\underline{\tau}}(f)$ are linear functions of $f$. By their uniqueness we obtain $b_{\underline{\tau}}\left(\phi_{\underline{\tau}^{\prime}}\right)=\delta_{\underline{\tau}, \tau^{\prime}}$. The coefficient functionals $\left\{b_{\underline{\tau}}\right\}$ form therefore a biorthogonal system to the diamond basis $\left\{\phi_{\underline{\tau}}\right\}$. This system is explicitly known and for given $f \in C\left(I^{d}\right)$ and $\underline{\tau} \in D^{d}$ the values of the corresponding 
functionals are given as follows (see [5]):

$$
b_{\underline{\tau}}(f)=f(\underline{\tau}) \quad \text { for } \underline{\tau} \in D_{0, d},
$$

and for $k \geq 1$,

$$
b_{\underline{\tau}}(f)=\frac{1}{2^{d}} \sum_{\varepsilon \in\{-1,1\}^{d}}\left(f(\underline{\tau})-f\left(\underline{\tau}^{\varepsilon}\right)\right) \quad \text { for } \underline{\tau} \in D_{k, d},
$$

where $\underline{\tau}^{\varepsilon}=\left(\underline{\tau}_{1}^{\varepsilon}, \ldots, \underline{\tau}_{d}^{\varepsilon}\right)$ with

$$
\tau_{i}^{\varepsilon}= \begin{cases}\tau_{i}+\varepsilon_{i} \cdot 2^{-k} & \text { if } \tau_{i} \in D_{k}, \\ \tau_{i} & \text { if } \tau_{i} \in C_{k-1} .\end{cases}
$$

It is convenient to introduce the following finite-dimensional projections in the space $C\left(I^{d}\right)$ :

$$
R_{k}(f)=\sum_{\underline{\tau} \in D_{k, d}} b_{\underline{\tau}}(f) \phi_{\underline{\tau}} .
$$

The fact that $\left(\phi_{\underline{\tau}}(\underline{t}): \underline{\tau} \in D^{d}\right)$ is an interpolating basis in $C\left(I^{d}\right)$ can now be stated as

Proposition 2.2. For each $f \in C\left(I^{d}\right)$ the series

$$
\sum_{k=0}^{\infty} R_{k}(f)
$$

converges to $f$ in the maximum norm $\|\cdot\|_{\infty}$. The $k$ th partial sum

$$
P_{k}(f)=\sum_{l=0}^{k} R_{l}(f)
$$

interpolates $f$ at the set $C_{k}^{d}$ of dyadic points, i.e. for each $k \geq 0$,

$$
f(\underline{\tau})=P_{k}(f)(\underline{\tau}) \quad \text { for } \underline{\tau} \in C_{k}^{d} .
$$

Consequently, for all $k$ and nonnegative $f$ from $C\left(I^{d}\right)$ we have $P_{k} f \geq 0$. Moreover the basis constant is 1, i.e. $\left\|P_{k} f\right\|_{\infty} \leq\|f\|_{\infty}$ for all $k$ and $f \in$ $C\left(I^{d}\right)$. One more property of the diamond basis functions should be mentioned here (see (2.2) in [2]).

Proposition 2.3. For any reals $b_{\underline{\tau}}$ and $1 \leq p \leq \infty$ we have

$$
\left\|\sum_{\underline{\tau} \in D_{k, d}} b_{\underline{\tau}} \phi_{\underline{\tau}}\right\|_{p} \sim 2^{-d k / p}\left(\sum_{\underline{\tau} \in D_{k, d}}\left|b_{\underline{\tau}}\right|^{p}\right)^{1 / p}
$$

where the implied constants depend only on the dimension d. In particular,

$$
\left\|\phi_{\underline{\tau}}\right\|_{p} \sim 2^{-d k / p} \quad \text { for } \underline{\tau} \in D_{k, d} .
$$

The main result motivating our algorithm in the diamond case was established in [2], Theorem 3.3. Before we state its particular case we recall 
that the inequality $d / p<\alpha$ implies the embedding $B_{p, q}^{\alpha} \subset C\left(I^{d}\right)$, so for $f \in B_{p, q}^{\alpha}$ the series (2.16) converges uniformly over $I^{d}$.

Theorem 2.4. Let $1 \leq p, q<\infty$ and $p / d<\alpha<1$. Then for $f \in B_{p, q}^{\alpha}$,

$$
\|f\|_{p, q}^{(\alpha)} \sim\left(\sum_{k=0}^{\infty}\left[2^{k(\alpha-d / p)}\left(\sum_{\underline{\tau} \in D_{k, d}}\left|b_{\underline{\underline{\tau}}}\right|^{p}\right)^{1 / p}\right]^{q}\right)^{1 / q},
$$

where the implied constants do not depend on $f$.

Corollary 2.5. Let $1 \leq p, q<\infty$ and $p / d<\alpha<1$. Then the system $\left\{\phi_{\underline{\tau}}\right\}$ defined in (2.15) is an unconditional basis in $B_{p, q}^{\alpha}$. Moreover, when normalized in $B_{p, q}^{\alpha}$, it is democratic if and only if $p=q$.

Proof. According to (2.26) we have

$$
\left\|\phi_{\underline{\tau}}\right\|_{p, q}^{(\alpha)} \sim 2^{k(\alpha-d / p)} \quad \text { for } \underline{\tau} \in D_{k, d} .
$$

Introducing $\phi_{\underline{\underline{\tau}} ; p, q}^{(\alpha)}=\phi_{\underline{\underline{\tau}}} /\left\|\phi_{\underline{\underline{\tau}}}\right\|_{p, q}^{(\alpha)}$ we can rewrite (2.16) as follows:

$$
f=\sum_{k=0}^{\infty} \sum_{\underline{\tau} \in D_{k, d}} a_{\underline{\tau}} \phi_{\underline{\tau} ; p, q}^{(\alpha)} .
$$

Now, combining (2.28), (2.27) and Theorem 2.4 we get

$$
\|f\|_{p, q}^{(\alpha)} \sim\left(\sum_{k=0}^{\infty}\left(\sum_{\tau \in D_{k, d}}\left|a_{\underline{\tau}}\right|^{p}\right)^{q / p}\right)^{1 / q} .
$$

Thus, the sequence space $\mathcal{A}_{p, q}$ of all $\underline{a}=\left\{a_{\underline{\tau}}: a_{\underline{\tau}} \in D_{k, d}, k \geq 0\right\}$ with the norm

$$
\|\underline{a}\|_{p, q}=\left(\sum_{k=0}^{\infty}\left(\sum_{\underline{\tau} \in D_{k, d}}\left|a_{\underline{\tau}}\right|^{p}\right)^{q / p}\right)^{1 / q}
$$

is linearly isomorphic to $\left[B_{p, q}^{\alpha},\|\cdot\|_{p, q}^{(\alpha)}\right]$. Since this isomorphism takes the normalized diamond basis into the customary unit basis vectors in $\mathcal{A}_{p, q}$, it is sufficient to check that the latter basis is democratic if and only if $p=q$. If $p=q$, then $\mathcal{A}_{p, p}$ is simply the $l_{p}$ space and the unit basis vectors form a democratic basis. Let us now take unit basis vectors $\underline{e}_{\underline{\tau}_{k}} \in \mathcal{A}_{p, q}$ with $\underline{\tau}_{k} \in D_{k, d}, k=1, \ldots, m$. Then

$$
\left\|\underline{e}_{\underline{\tau}_{1}}+\ldots+\underline{e}_{\underline{\tau}_{m}}\right\|_{p, q} \sim m^{1 / q}
$$

and if for fixed $k$ we are given $m$ unit basis vectors $\underline{e}_{\underline{\tau}_{i}^{*}} \in \mathcal{A}_{p, q}$ with $\underline{\tau}_{i}^{*} \in D_{k, d}$, $i=1, \ldots, m$, then

$$
\left\|\underline{e}_{\underline{\tau}_{1}^{*}}+\ldots+\underline{e}_{\underline{\tau}_{m}^{*}}\right\|_{p, q} \sim m^{1 / p}
$$

Comparing (2.31) and (2.32) leads to the conclusion that $p=q$ and this completes the proof. 
Corollary 2.6. Let $1 \leq p<\infty$ and $p / d<\alpha<1$. Then as a model space for the diamond compression we can take the Besov space $B_{p, p}^{\alpha}$ with the basis $\phi_{\underline{\tau} ; p, p}^{(\alpha)}$, which is greedy.

We now turn to outlining the corresponding properties of the Haar orthogonal system over $I^{d}$, in which case it is asumed that $I=[0,1)$. Let us start with the definition of the Haar functions over $I^{d}$. We begin with the following decompositions:

$$
I=\bigcup_{k=0}^{2^{j-1}-1} I_{j, k} \quad \text { for } j \geq 1
$$

where

$$
I_{j, k}=\left[\frac{k}{2^{j-1}}, \frac{k+1}{2^{j-1}}\right), \quad\left|I_{j, k}\right|=\frac{1}{2^{j-1}} .
$$

Observe that this leads for each $j \geq 1$ to the splitting formula

$$
I_{j, k}=I_{j+1,2 k} \cup I_{j+1,2 k+1} \quad \text { for } k=0, \ldots, 2^{j-1}-1 .
$$

Definition 2.1. The Haar functions over $I$ are defined as follows:

$$
h_{0}(t)=1 \quad \text { for } t \in I,
$$

and for $0 \leq k<2^{j-1}, j \geq 1$,

$$
h_{2^{j-1}+k}(t)=h_{j, k}(t)= \begin{cases}1 & \text { for } t \in I_{j+1,2 k}, \\ -1 & \text { for } t \in I_{j+1,2 k+1}, \\ 0 & \text { elsewhere in } I .\end{cases}
$$

It now follows that

$$
h_{j, k}(t)=h_{1}\left(2^{j-1} t-k\right) .
$$

Now one checks directly the orthogonality of the system

$$
\left\{h_{n}: n \geq 0\right\}
$$

with respect to the Lebesgue measure on $I$. We mention only that the Haar system (2.39) is an orthogonal basis in $L^{p}(I)$ for $1 \leq p<\infty$ (see [6]). For later convenience we introduce $\mathbb{Z}_{+}=\{k \in \mathbb{Z}: k \geq 0\}, D=\{1, \ldots, d\}$, and for $\underline{k}=\left(k_{1}, \ldots, k_{d}\right)$ define $\vee \underline{k}=\max \left(k_{1}, \ldots, k_{d}\right)$. The multivariate Haar orthogonal system will be indexed by elements of $\mathbb{Z}_{+}^{d}$ according to the decomposition

$$
\mathbb{Z}_{+}^{d}=\bigcup_{j \in \mathbb{Z}_{+}} Z_{j, d}
$$

where

$$
Z_{j, d}= \begin{cases}\left\{\underline{k} \in \mathbb{Z}_{+}^{d}: 2^{j-1} \leq \vee \underline{k}<2^{j}\right\} & \text { for } j>0 \\ \underline{0} \in \mathbb{Z}_{+}^{d} & \text { for } j=0 .\end{cases}
$$


We also introduce

$$
Y_{n, d}=\bigcup_{j=0}^{n} Z_{j, d} \quad \text { for } \quad n \in \mathbb{Z}_{+} .
$$

Definition 2.2. The Haar function over $I^{d}$ with index $\underline{k} \in Z_{j, d}$ is defined as follows: If $\underline{k}=\underline{0}$, then

$$
h_{\underline{0}}=\bigotimes_{i=1}^{d} h_{0} .
$$

If $\underline{k} \in Z_{j, d}$ and $E=\left\{i \in D: 2^{j-1} \leq k_{i}<2^{j}\right\}$, then

$$
h_{\underline{k}}=\bigotimes_{i \in E} h_{k_{i}} \otimes \bigotimes_{i \in D \backslash E}\left|h_{2^{j-1}+k_{i}}\right| .
$$

COROLlary 2.7. The Haar system $\left\{h_{\underline{k}}: \underline{k} \in \mathbb{Z}_{+}^{d}\right\}$ is orthogonal and for $1 \leq p \leq \infty$ and real $\left\{a_{\underline{k}} \in \mathbb{R}: \underline{k} \in Z_{j, d}\right\}$ we have, for $j \geq 0$,

$$
\left\|\sum_{\underline{k} \in Z_{j, d}} a_{\underline{k}} h_{\underline{k}}\right\|_{p} \sim\left(2^{-j d} \sum_{\underline{k} \in Z_{j, d}}\left|a_{\underline{k}}\right|^{p}\right)^{1 / p},
$$

where the implied constants depend only on $d$. In particular, for $\underline{k} \in Z_{j, d}$,

$$
\left\|h_{\underline{k}}\right\|_{p} \sim 2^{-j d / p} .
$$

To the index sets (2.41) and (2.42) there correspond respectively the finite-dimensional function spaces

$$
\begin{aligned}
W_{j} & =\operatorname{span}\left\{h_{\underline{k}}: \underline{k} \in Z_{j, d}\right\}, \\
V_{n} & =\operatorname{span}\left\{h_{\underline{k}}: \underline{k} \in Y_{n, d}\right\} .
\end{aligned}
$$

We introduce the respective orthogonal projections of $L^{2}\left(I^{d}\right)$ onto $W_{j}$ and onto $V_{n}$ :

$$
\begin{aligned}
& Q_{j}(f)=\sum_{\underline{k} \in Z_{j, d}}\left(f, h_{\underline{k}}\right) h_{\underline{k}} /\left\|h_{\underline{k}}\right\|_{2}^{2}, \\
& P_{n}(f)=\sum_{\underline{k} \in Y_{n, d}}\left(f, h_{\underline{k}}\right) h_{\underline{k}} /\left\|h_{\underline{k}}\right\|_{2}^{2} .
\end{aligned}
$$

Lemma 2.8. For $f \in L^{p}\left(I^{d}\right)$ and $1 \leq p<\infty$ we have the Schauder decomposition

$$
f=\sum_{j \in \mathbb{Z}_{+}} Q_{j}(f) .
$$

For the partial sum $P_{n}(f)$ of $f \in L^{1}$ we have the formula

$$
P_{n}(f)(x)=\frac{1}{|Q|} \int_{Q} f(y) d y \quad \text { for } x \in Q,
$$


where $Q \subset I^{d}$ is a dyadic cube of volume $2^{-d n}$. Moreover, for $f \in L^{p}$, $1 \leq p \leq \infty$ and $n \in \mathbb{Z}_{+}$we have

$$
\begin{gathered}
\left\|P_{n}(f)\right\|_{p} \leq\|f\|_{p}, \\
\left\|f-P_{n}(f)\right\|_{p} \leq C \omega_{p}\left(f ; 2^{-n}\right) .
\end{gathered}
$$

The following Bernstein type inequality also holds:

$$
\omega_{p}(f ; \delta) \leq C\left(\min \left(\delta \cdot 2^{n}, 1\right)\right)^{1 / p}\|f\|_{p} \quad \text { for } f \in V_{n},
$$

where $C$ depends only on $d$.

Proof. Most of the statements are well known at least in one variable. Extension to several variables for the Haar system defined as in (2.43) and (2.44) is straightforward.

TheOREM 2.9. For $f \in L^{1}\left(I^{d}\right)$ we have the following Fourier-Haar expansion convergent in $L^{1}$ :

$$
f=\sum_{j \in \mathbb{Z}_{+}} \sum_{\underline{k} \in Z_{j, d}} b_{\underline{k}} h_{\underline{k}},
$$

where

$$
b_{\underline{k}}=\left(f, h_{\underline{k}}\right) /\left\|h_{\underline{k}}\right\|_{2}^{2} .
$$

Let now $1 \leq p, q<\infty$ and $0<\alpha<1 / p \leq 1$. Then for $f \in B_{p, q}^{\alpha}$ we have

$$
\|f\|_{p, q}^{(\alpha)} \sim\left(\sum_{j \in \mathbb{Z}_{+}}\left[2^{j(\alpha-d / p)}\left(\sum_{\underline{k} \in Z_{j, d}}\left|b_{\underline{k}}\right|^{p}\right)^{1 / p}\right]^{q}\right)^{1 / q},
$$

where the implied constants do not depend on $f$.

Proof. Using Lemma 2.8 we can prove by standard arguments the equivalence

$$
\left(\sum_{n \in \mathbb{Z}_{+}}\left(2^{\alpha n} \omega_{p}\left(f ; 1 / 2^{n}\right)\right)^{q}\right)^{1 / q} \sim\left(\sum_{n \in \mathbb{Z}_{+}}\left(2^{\alpha n}\left\|Q_{n} f\right\|_{p}\right)^{q}\right)^{1 / q},
$$

and consequently by Corollary 2.7 we obtain (2.58).

COROLlary 2.10. The Haar functions $h_{\underline{k} ; p, q}^{(\alpha)}$ normalized in $B_{p, q}^{\alpha}, 0<$ $\alpha<1 / p, 1 \leq p, q<\infty$, form a greedy basis in this space if and only if $p=q$. Thus, we can take $B_{p, p}^{\alpha}\left(I^{d}\right)$ with $0<\alpha<1 / p$ as a model space for the Haar compression.

3. The diamond compression. We start with a fixed power of two, $2^{n}, n \geq 0$, and the interval of integers $J=\left[0, \ldots, 2^{n}\right]$. Denote by $L_{n}(J)$ the real vector space of all real vectors $\mathbf{x}=\{x(k): k \in J\}$; the dimension of $L_{n}(J)$ is $|J|=2^{n}+1$ (here and in what follows, the number of elements 
of a finite set $A$ is denoted by $|A|$ ). There is a natural basis of unit vectors in $L_{n}(J)$ :

$$
\mathbf{e}_{i}=\left\{e_{i}(k): k \in J\right\} \quad \text { with } e_{i}(k)=\delta_{i, k} \text { for } i, k \in J .
$$

For each $\mathbf{x} \in L_{n}(J)$ we have the basis representation

$$
\mathbf{x}=\sum_{i \in J} x(i) \mathbf{e}_{i}
$$

Now, we are going to define the one-dimensional discrete Schauder basis and the corresponding partitions of unity. We use the function

$$
\psi(t)=\max [0,1-|t|] \quad \text { for } t \in \mathbb{R} .
$$

Now, each $i \in J$ is of the form

$$
i=2^{n-j} \cdot \nu
$$

where the integer $n-j$ is the maximal power of 2 dividing $i$; here $\nu$ is odd for $i \neq 0$, with $0<\nu<2^{j}$, and for $i=0$ we set $j=0, \nu=0$. Now, for each $i \in J$ represented as in (3.4), the corresponding Schauder function over $J$ is defined as follows:

$$
\phi_{i}(m)=\psi\left(2^{j-n} m-\nu\right) \quad \text { for } m \in J,
$$

The Schauder functions are linearly independent and

$$
L_{n}(J)=\operatorname{span}\left\{\underline{\phi}_{i}: i \in J\right\} .
$$

Thus, for each $\mathbf{x} \in L_{n}(J)$ there is a unique $\mathbf{a} \in L_{n}(J)$ such that

$$
\mathbf{x}=\sum_{i \in J} a(i) \underline{\phi}_{i}
$$

Now, it is known and also easy to check that for $i$ as in (3.4),

$$
a(i)= \begin{cases}x(i) & \text { for } i=0,2^{n} \\ x(i)-\frac{1}{2}\left[x\left(i-2^{n-j}\right)+x\left(i+2^{n-j}\right)\right] & \text { for } 0<i<2^{n} .\end{cases}
$$

A system of functions in $L_{n}(J)$ is called a partition of unity if its members are nonnegative and add up to the constant function 1 . We now define a family of such partitions. For given $s, 0 \leq s \leq n$, and $\nu, 0 \leq \nu \leq 2^{s}$, define

$$
\psi_{s, \nu}(m)=\psi\left(2^{s-n} m-\nu\right) \quad \text { for } m \in J .
$$

It follows that in vector notation

$$
\sum_{\nu=0}^{2^{s}} \underline{\psi}_{s, \nu}=\mathbf{1}
$$

In particular, in the extreme cases of $s=0$ and $s=n$ we obtain respectively $\underline{\psi}_{0,0}=\underline{\phi}_{0}, \underline{\psi}_{0,1}=\underline{\phi}_{2^{n}}$ and $\underline{\psi}_{n, \nu}=\mathbf{e}_{\nu}$ for $\nu \in J$. Formula (3.9) also yields

$$
\underline{\phi}_{i}=\underline{\psi}_{j, \nu} \quad \text { for } i \text { as in (3.4). }
$$


Let us now introduce an increasing sequence of subspaces of $L_{n}(J)$ by setting

$$
L_{n, s}(J)=\operatorname{span}\left\{\underline{\psi}_{s, \nu}: 0 \leq \nu \leq 2^{s}\right\} \quad \text { with } 0 \leq s \leq n .
$$

Clearly, $\left\{\underline{\psi}_{s, \nu}: 0 \leq \nu \leq 2^{s}\right\}$ is a basis in $L_{n, s}(J)$ and $L_{n, n}(J)=L_{n}(J)$. However, in the same space there is another basis, namely a suitable part of the Schauder basis. More precisely, using (3.11), we get

$$
L_{n, s}(J)=\operatorname{span}\left\{\underline{\phi}_{i}=\underline{\psi}_{j, \nu}: 0 \leq j \leq s \text { and } \nu \text { as in (3.4) }\right\} .
$$

Let now $0<s \leq n$. Then the basis $\left\{\underline{\psi}_{s, \nu}: 0 \leq \nu \leq 2^{s}\right\}$ in $L_{n, s}(J)$ is interpolating at the points $m$ for which $\underline{\psi}_{s, \nu}(m)=1$. Consequently, for $\mathbf{y} \in L_{n, s}(J)$ we have

$$
\mathbf{y}=\sum_{\nu=0}^{2^{s}} y\left(2^{n-s} \nu\right) \underline{\psi}_{s, \nu} .
$$

Applying this to $\mathbf{y}=\underline{\psi}_{s-1, \mu}$ and using (3.9) we get the lifting up formula

$$
\underline{\psi}_{s-1, \mu}=\underline{\psi}_{s, 2 \mu}+\frac{\underline{\psi}_{s, 2 \mu-1}+\underline{\psi}_{s, 2 \mu+1}}{2} .
$$

We now turn to the multidimensional case. As in one dimension, $L_{n}\left(J^{d}\right)$ denotes the real vector space of real vectors $\mathbf{x}=\left\{x(\underline{m}): \underline{m} \in J^{d}\right\}$. We also introduce an increasing sequence of its subspaces by setting

$$
L_{n, s}\left(J^{d}\right)=\operatorname{span}\left\{\underline{\psi}_{s, \underline{\nu}}: \underline{\nu} \in J^{d}, \vee \underline{\nu} \leq 2^{s}\right\} \quad \text { with } 0 \leq s \leq n,
$$

where

$$
\underline{\psi}_{s, \underline{\mu}}=\bigotimes_{k=1}^{d} \underline{\psi}_{s, \mu_{k}} .
$$

The system $\left\{\underline{\psi}_{s, \underline{\nu}}: \underline{\nu} \in J^{d}, \vee \underline{\nu} \leq 2^{s}\right\}$ is a partition of unity and it is a basis in $L_{n, s}\left(J^{d}\right)$. As in the one-dimensional case we have

Proposition 3.1. For $s=1, \ldots, n$ and $\left\{\underline{\mu} \in J^{d}: \vee \underline{\mu} \leq 2^{s-1}\right\}$ we have

$$
\underline{\psi}_{s-1, \underline{\mu}}=\sum_{\underline{\varepsilon} \in\{-1,0,1\}^{d}} \frac{1}{2^{|\underline{\varepsilon}|}} \underline{\psi}_{s, 2 \underline{\mu}+\underline{\varepsilon}},
$$

where $|\underline{\varepsilon}|=\left|\varepsilon_{1}\right|+\ldots+\left|\varepsilon_{d}\right|$.

Proof. Take $\mathbf{u} \in L_{n, s}\left(J^{d}\right)$. Since the basis $\left\{\underline{\psi}_{s, \underline{\nu}}: \underline{\nu} \in J^{d}, \vee \underline{\nu} \leq 2^{s}\right\}$ is interpolating at the points $2^{n-s} \underline{\nu}$ it follows that

$$
\mathbf{u}=\sum_{\vee \underline{\nu} \leq 2^{s}} u\left(2^{n-s} \underline{\nu}\right) \underline{\psi}, \underline{\nu} .
$$


In particular, since $\underline{\psi}_{s-1, \underline{\nu}} \in L_{n, s}\left(J^{d}\right)$, it follows from (3.19) that

$$
\underline{\psi}_{s-1, \underline{\mu}}=\sum_{\underline{V}_{\underline{\nu} \leq 2^{s}}} \underline{\psi}_{s-1, \underline{\mu}}\left(2^{n-s} \underline{\nu}\right) \underline{\psi}_{s, \underline{\nu}} .
$$

Now, (3.20), (3.17), and (3.9) imply (3.18).

To define the diamond basis in $L_{n}\left(J^{d}\right)$ we need the following decomposition of the index set $J$ :

$$
J=\bigcup_{j=0}^{n} \delta(J)_{j}
$$

where $\delta(J)_{0}=\left\{0,2^{n}\right\}$ and

$$
\delta(J)_{j}=\left\{2^{n-j} \nu: 1 \leq \nu \leq 2^{j}, \nu \text { odd }\right\} \quad \text { for } 1 \leq j \leq n .
$$

We also introduce $J_{0}=\left\{0,2^{n}\right\}, J_{j}=J_{j-1} \cup \delta(J)_{j}$. Clearly, $J_{n}=J$. Hence,

$$
J_{j}=\left\{2^{n-j} \nu: 0 \leq \nu \leq 2^{j}\right\} \quad \text { for } j=1, \ldots, n .
$$

We may now decompose the $d$-dimensional index set $J^{d}$. Define

$$
\delta\left(J^{d}\right)_{0}=J_{0}^{d}, \quad \delta\left(J^{d}\right)_{j}=J_{j}^{d} \backslash J_{j-1}^{d} \quad \text { for } j=1, \ldots, n .
$$

Then

$$
\begin{aligned}
\delta\left(J^{d}\right)_{j} & =\left\{\underline{i} \in J_{j}^{d}: \exists_{k} i_{k} \in \delta(J)_{j}\right\}, \\
J^{d} & =\bigcup_{j=0}^{n} \delta\left(J^{d}\right)_{j} .
\end{aligned}
$$

Definition 3.1. For $\underline{i} \in \delta\left(J^{d}\right)_{j}, 0 \leq j \leq n$, define

$$
\underline{\phi_{i}}=\bigotimes_{k=1}^{d} \underline{\psi}_{j, \nu_{k}} \quad \text { with } \quad \nu_{k}=i_{k} 2^{j-n} .
$$

In this formula, if $\nu_{k}$ is odd, then $\underline{\psi}_{j, \nu_{k}}=\underline{\phi}_{i_{k}}$ with $i_{k}=2^{n-j} \nu_{k}$. The system $\left\{\underline{\phi_{i}}: \underline{i} \in J^{d}\right\}$ is a basis in $L_{n}\left(J^{d}\right)$, called the diamond or multi-affine basis.

Thus, for each $\mathbf{y} \in L_{n}\left(J^{d}\right)$ there is a unique $\mathbf{b} \in L_{n}\left(J^{d}\right)$ such that

$$
\mathbf{y}=\sum_{\underline{i} \in J^{d}} b(\underline{i}) \underline{\phi} .
$$

Definition 3.2. The map $\mathbf{S}: \mathbf{y} \mapsto \mathbf{b}$ is called the Schauder transform.

Our goal is to describe algorithms for $\mathbf{S}$ and for its inverse, i.e. for calculating $\mathbf{b}$ in terms of $\mathbf{y}$ and vice versa. Extension of (3.8) to the $d$-dimensional case solves the first part. Such a formula is known [5]:

Proposition 3.2. If $j=0$, then

$$
b(\underline{i})=y(\underline{i}) \quad \text { for } \underline{i} \in \delta\left(J^{d}\right)_{j},
$$


and if $1 \leq j \leq n$, then

$$
b(\underline{i})=y(\underline{i})-\frac{1}{2^{d}} \sum_{\underline{\varepsilon} \in\{-1,1\}^{d}} y\left(\underline{i}+2^{n-j} \underline{\varepsilon}^{*}\right) \quad \text { for } \underline{i} \in \delta\left(J^{d}\right)_{j},
$$

where $\underline{\varepsilon}^{*}$ is defined as follows: Since $i_{k} \in J_{j}$ it follows by (3.23) that $i_{k}=$ $2^{n-j} \nu_{k}$ with $0 \leq \nu_{k} \leq 2^{j}$. Now, put

Moreover,

$$
\varepsilon_{k}^{*}= \begin{cases}0 & \text { if } \nu_{k} \text { is even }, \\ \varepsilon_{k} & \text { if } \nu_{k} \text { is odd. }\end{cases}
$$

$$
|b(\underline{i})| \leq 2 \max \left\{\left|y\left(\underline{i}^{\prime}\right)\right|: \underline{i}^{\prime} \in J^{d}\right\} \quad \text { for } \underline{i} \in J^{d},
$$

and for $\mathbf{y} \geq 0$,

$$
|b(\underline{i})| \leq \max \left\{y\left(\underline{i}^{\prime}\right): \underline{i}^{\prime} \in J^{d}\right\} \quad \text { for } \underline{i} \in J^{d} .
$$

Now we are interested in finding an algorithm for $\mathbf{S}^{-1}$. Given $\mathbf{b}$ in formula (3.28) we want to recover $\mathbf{y}$. Using decomposition (3.26) we rewrite (3.28) as

$$
\mathbf{y}=\sum_{j=0}^{n} \sum_{\delta\left(J^{d}\right)_{j}} b(\underline{i}) \underline{\phi} \underline{.} .
$$

In addition we introduce the corresponding partial sums

$$
\mathbf{y}^{(s)}=\sum_{j=0}^{s} \sum_{\delta\left(J^{d}\right)_{j}} b(\underline{i}) \underline{\phi_{i}} .
$$

Since $\mathbf{y}^{(s)} \in L_{n, s}\left(J^{d}\right)$, by (3.16) we have a unique representation

$$
\mathbf{y}^{(s)}=\sum_{\vee \underline{\nu} \leq 2^{s}} b^{(s)}(\underline{\nu}) \underline{\psi}_{s, \underline{\nu}} .
$$

The basic algorithm for calculating the inverse to $\mathbf{S}$ is presented in

Proposition 3.3. For the vectors $\mathbf{b}^{(s)}=\left\{b^{(s)}(\underline{\mu}): \vee \underline{\mu} \leq 2^{s}\right\}$ with $s=$ $0, \ldots, n$, given $\mathbf{b}$, we have the following recurrence relation:

$$
b^{(0)}(\underline{\mu})=b\left(2^{n} \underline{\mu}\right) \quad \text { for } \underline{\mu} \in\{0,1\}^{d},
$$

and for $s=1, \ldots, n$ and $\vee \underline{\mu} \leq 2^{s}$,

$$
b^{(s)}(\underline{\mu})=b_{1}^{(s)}(\underline{\mu})+b_{2}^{(s)}(\underline{\mu}),
$$

where

$$
\begin{aligned}
& b_{1}^{(s)}(\underline{\mu})=\sum_{\underline{\varepsilon} \in\{-1,0,1\}^{d}} \frac{1}{2^{|\underline{\varepsilon}|}} b^{(s-1)}\left(\left\lfloor\underline{\underline{\mu}+\underline{1}-\underline{\varepsilon}} \frac{}{2}\right\rfloor,\right. \\
& b_{2}^{(s)}(\underline{\mu})= \begin{cases}b\left(2^{n-s} \underline{\mu}\right) & \text { if } 2^{s-1}<\vee \underline{\mu} \leq 2^{s}, \\
0 & \text { if } \vee \underline{\mu} \leq 2^{s-1} .\end{cases}
\end{aligned}
$$


Proof. We start with the relation

$$
\mathbf{y}^{(s)}=\mathbf{y}^{(s-1)}+\sum_{\delta\left(J^{d}\right)_{s}} b(\underline{i}) \underline{\phi_{i}} .
$$

We rewrite both terms on the right hand side of (3.40) in the same basis as in (3.35). Using Proposition 3.1 we obtain

$$
\begin{aligned}
\mathbf{y}^{(s-1)} & =\sum_{\vee \underline{\nu} \leq 2^{s-1}} b^{(s-1)}(\underline{\nu}) \underline{\psi}_{s-1, \underline{\nu}} \\
& =\sum_{\vee \underline{\nu} \leq 2^{s-1}} b^{(s-1)}(\underline{\nu}) \sum_{\underline{\varepsilon} \in\{-1,0,1\}^{d}} \frac{1}{2^{|\underline{\varepsilon}|}} \underline{\psi}_{s, 2 \underline{\nu}+\underline{\varepsilon}} \\
& =\sum_{\underline{\varepsilon} \in\{-1,0,1\}^{d}} \frac{1}{2^{|\underline{\varepsilon}|}} \sum_{\vee \underline{\nu} \leq 2^{s-1}} b^{(s-1)}(\underline{\nu}) \underline{\psi}_{s, 2 \underline{\nu}+\underline{\varepsilon}} \\
& =\sum_{\underline{\varepsilon} \in\{-1,0,1\}^{d}} \frac{1}{2^{|\underline{\varepsilon}|}} \sum_{\vee \underline{\nu} \leq 2^{s-1}}^{\prime} b^{(s-1)}(\underline{\nu}) \underline{\psi}_{s, 2 \underline{\nu}+\underline{\varepsilon}},
\end{aligned}
$$

where the last summation is taken over all admissible $\underline{\nu}$, i.e. such that $0 \leq$ $2 \nu_{k}+\varepsilon_{k} \leq 2^{s}$ for $k=1, \ldots, d$. Now, given $\underline{\varepsilon}$, for each $\underline{\mu}$ with $\vee \underline{\mu} \leq 2^{s}$, there is a unique $\underline{\nu}$ with $\vee \underline{\nu} \leq 2^{s-1}$ such that $\underline{\mu}=2 \underline{\nu}+\underline{\varepsilon}$ and $\underline{\nu}=\lfloor(\bar{\mu}+\underline{1}-\underline{\varepsilon}) / 2\rfloor$ $(\lfloor x\rfloor$ is the integer part of $x)$. Consequently,

$$
\mathbf{y}^{(s-1)}=\sum_{\vee \underline{\mu} \leq 2^{s}}\left(\sum_{\underline{\varepsilon} \in\{-1,0,1\}^{d}} \frac{1}{2^{|\underline{\varepsilon}|}} b^{(s-1)}\left(\left\lfloor\underline{\underline{\mu}+\underline{1}-\underline{\varepsilon}} \frac{\mid}{2}\right\rfloor\right) \underline{\psi}_{s, \underline{\mu}} .\right.
$$

To represent the second term of the right hand side of (3.40) we use Definition 3.1: for $\underline{i} \in \delta\left(J^{d}\right)_{s}, 1 \leq s \leq n$, we have

$$
\underline{\phi}_{i}=\underline{\psi}_{s, \underline{\mu}} \quad \text { with } \quad 2^{n-1} \underline{\mu}=\underline{i} \text {. }
$$

Thus,

$$
\sum_{\delta\left(J^{d}\right)_{s}} b(\underline{i}) \underline{\phi}_{\underline{i}}=\sum_{\delta\left(J^{d}\right)_{s}} b(\underline{i}) \underline{\psi}_{s, \underline{\mu}}=\sum_{2^{s-1}<\bigvee \underline{\mu} \leq 2^{s}} b\left(2^{n-s} \underline{\mu}\right) \underline{\psi}_{s, \underline{\mu}} .
$$

Now, (3.41) and (3.42) imply (3.37)-(3.39) and the proof is complete.

Corollary 3.4. If $\mathbf{b}$ is the Schauder transform of the image $\mathbf{y}$, then $\mathbf{y}^{(0)}, \mathbf{y}^{(1)}, \ldots, \mathbf{y}^{(n)}$ are consecutive approximations (recovers) of $\mathbf{y}$ in terms of $\mathbf{b}$. In particular $\mathbf{y}=\mathbf{y}^{(n)}$. It also follows from the algorithm that to calculate $\mathbf{y}^{(s)}$ we need to know $b(\underline{i})$ only for $\underline{i} \in J_{s}^{d}$.

For theoretical reasons presented in Section 2, in the diamond case it is natural to consider the following Besov norm on $L_{n}\left(J^{d}\right)$ : for $\mathbf{y}$ as in (3.33), 
we define

$$
\|\mathbf{y}\|_{p}^{(\alpha)}=\left(\sum_{j=0}^{n} 2^{j(\alpha p-d)} \sum_{\underline{i} \in \delta\left(J^{d}\right)_{j}}|b(\underline{i})|^{p}\right)^{1 / p}
$$

with the admissible parameters $1 \leq p \leq \infty, d / p<\alpha<1$. In particular, if $\mathbf{y}=\phi_{\underline{i}}$ and $\underline{i} \in \delta\left(J^{d}\right)_{j}$, then $\left\|\phi_{\underline{i}}\right\|_{p}^{(\alpha)}=2^{j(\alpha-d / p)}$.

Definition 3.3. For the diamond functions normalized in the norm (3.43) we introduce the notation

$$
\underline{\phi}_{\underline{i}}^{(\alpha, p)}=2^{j(d / p-\alpha)} \underline{\phi}_{i} \quad \text { for } \underline{i} \in \delta\left(J^{d}\right)_{j}, 0 \leq j \leq n .
$$

COROLlary 3.5. If $\mathbf{y} \in L_{n}\left(J^{d}\right)$ is represented in the normalized diamond basis as given in (3.44), i.e.

$$
\mathbf{y}=\sum_{j=0}^{n} \sum_{\underline{i} \in \delta\left(J^{d}\right)_{j}} a(\underline{i}) \underline{\phi}_{i}^{(\alpha, p)}=\sum_{\underline{i} \in J^{d}} a(\underline{i}) \underline{\phi}_{i}^{(\alpha, p)},
$$

then

$$
\|\mathbf{y}\|_{p}^{(\alpha)}=\left(\sum_{j=0}^{n} \sum_{\underline{i} \in \delta\left(J^{d}\right)_{j}}|a(\underline{i})|^{p}\right)^{1 / p}=\left(\sum_{\underline{i} \in J^{d}}|a(\underline{i})|^{p}\right)^{1 / p},
$$

where

$$
a(\underline{i})=2^{j(\alpha-d / p)} b(\underline{i}) \quad \text { for } \underline{i} \in \delta\left(J^{d}\right)_{j} .
$$

Clearly, the basis $\left\{\underline{\phi}_{\underline{i}}^{(\alpha, p)}: \underline{i} \in J^{d}\right\}$ in the Banach space $\left[L_{n}\left(J^{d}\right),\|\cdot\|_{p}^{(\alpha)}\right]$ is greedy.

In order to have a way of dropping the irrelevant coefficients in the representation (3.45) we introduce the distribution function for the coefficients $\{a(\underline{i})\}$ which is related to thresholding. We recall the restrictions on the parameters $\alpha$ and $p$ : $1 \leq p \leq \infty, d / p<\alpha<1$. Now, introduce

$$
B(\lambda)=\left\{\underline{i} \in J^{d}:|a(\underline{i})| \leq \lambda\right\}, \quad \lambda \geq 0,
$$

and the distribution function

$$
F(\lambda)=|B(\lambda)|
$$

For given $\alpha, p$ and $\lambda$ we assign to $\mathbf{y}$ given by (3.45) the compressed $\mathbf{y}$ :

$$
\mathbf{y}_{\lambda}=\sum_{\underline{i} \in J^{d}} a_{\lambda}(\underline{i}) \underline{\phi}^{(\alpha, p)}
$$

where

$$
a_{\lambda}(\underline{i})= \begin{cases}a(\underline{i}) & \text { for } \underline{i} \in J^{d} \backslash B(\lambda), \\ 0 & \text { for } \underline{i} \in B(\lambda) .\end{cases}
$$


Proposition 3.6. The Besov distance between $\mathbf{y}$ and its compression $\mathbf{y}_{\lambda}$ can be estimated as follows:

$$
\left\|\mathbf{y}-\mathbf{y}_{\lambda}\right\|_{p}^{(\alpha)} \leq \lambda[F(\lambda)]^{1 / p}
$$

Equality in (3.52) is attained for any $\mathbf{y}$ of the form

$$
\sum_{\underline{i} \in J^{d}} \pm \lambda \underline{\phi}^{(\alpha, p)} \text {. }
$$

The proof of Proposition 3.6 is elementary. We only mention that the right hand side of (3.52) is a nondecreasing right-continuous piecewise linear function of $\lambda$ and it goes to zero as $\lambda \rightarrow 0$.

Definition 3.4. We say that $\mathbf{y}_{\lambda}$ is an $\varepsilon$-compression if

$$
\left\|\mathbf{y}-\mathbf{y}_{\lambda}\right\|_{p}^{(\alpha)} \leq \varepsilon .
$$

From the above we get

Corollary 3.7. If $\lambda[F(\lambda)]^{1 / p} \leq \varepsilon$, then $\mathbf{y}_{\lambda}$ is an $\varepsilon$-compression.

4. The Haar compression. As in the diamond case we start with a fixed power $2^{n}, n \geq 0$, and for technical reasons with the "semi-closed" interval of integers $I=\left[0, \ldots, 2^{n}\right)=\left[0, \ldots, 2^{n}-1\right]$; again $L_{n}(I)$ is a real vector space of dimension $|I|=2^{n}$. There is a natural basis of unit vectors in $L_{n}(I)$ :

$$
\mathbf{e}_{i}=\left\{e_{i}(k): k \in I\right\} \quad \text { with } e_{i}(k)=\delta_{i, k} \text { for } i, k \in I .
$$

In the natural scalar product in $L_{n}(I)$ :

$$
(\mathbf{x}, \mathbf{y})=\frac{1}{|I|} \sum_{k \in I} x(k) y(k),
$$

the basis (4.1) is orthogonal, i.e.

$$
\left(\mathbf{e}_{i}, \mathbf{e}_{k}\right)=\frac{\delta_{i, k}}{|I|} \quad \text { for } i, k \in I .
$$

Now, for each $\mathbf{x} \in L_{n}(I)$ we have the basic representation

$$
\mathbf{x}=\sum_{i \in I}\left(\mathbf{x}, \mathbf{e}_{i}\right) \mathbf{e}_{i}=\sum_{i \in I} x(i) \mathbf{e}_{i} .
$$

Along with $\left\{\mathbf{e}_{i}: i \in I\right\}$ we are going to consider the discrete Haar orthogonal basis and a system of partitions of unity. We start with the following decomposition of the index set $I$ :

$$
I=\bigcup_{k=0}^{2^{j}-1} I_{j, k} \quad \text { for } j=0, \ldots, n,
$$


where

$$
I_{j, k}=\left[2^{n-j} k, 2^{n-j}(k+1)\right), \quad\left|I_{j, k}\right|=2^{n-j} .
$$

Observe that this leads, for each $j, 0 \leq j<n$, to the splitting formula

$$
I_{j, k}=I_{j+1,2 k} \cup I_{j+1,2 k+1} \quad \text { for } k=0, \ldots, 2^{j}-1 .
$$

The Haar orthogonal basis is now defined as follows:

$$
\mathbf{h}_{0}=\mathbf{1} \text {, i.e. } h_{0}(i)=1 \text { for } i \in \mathbb{Z} \text {, }
$$

and for $m=2^{j}+k$ with $0 \leq k<2^{j}$ and $0 \leq j<n$ define $\mathbf{h}_{m}$ as follows:

$$
h_{j, k}(i)=h_{m}(i)= \begin{cases}1 & \text { for } i \in I_{j+1,2 k}, \\ -1 & \text { for } i \in I_{j+1,2 k+1}, \\ 0 & \text { elsewhere in } \mathbb{Z},\end{cases}
$$

where $\mathbb{Z}$ is the set of all integers. It now follows that

$$
h_{2^{j}+k}(i)=h_{1}\left(2^{j} i-k 2^{n}\right) \quad \text { for } i \in I .
$$

Now one checks directly the orthogonality formula

$$
\left(\mathbf{h}_{m}, \mathbf{h}_{m^{\prime}}\right)=\delta_{m, m^{\prime}} \frac{\left|\operatorname{supp} \mathbf{h}_{m}\right|}{|I|} \text { for } m, m^{\prime} \in I \text {. }
$$

Notice that $\operatorname{supp} \mathbf{h}_{0}=I$ and $\operatorname{supp} \mathbf{h}_{m}=I_{j, k}$ for $m=2^{j}+k$.

Definition 4.1. The system $\left\{\mathbf{h}_{m}: m \in I\right\}$ is called the discrete Haar orthogonal basis in $L_{N}(I)$. It is normalized so that $\left(\mathbf{h}_{j, k}, \mathbf{h}_{j, k}\right)=2^{-j}$ or equivalently that $\max \left|\mathbf{h}_{j, k}\right|=1$.

Consequently, for each $\mathbf{x} \in L_{n}(I)$ we have the representation

$$
\mathbf{x}=\sum_{k \in I} b_{k} \mathbf{h}_{k} \quad \text { with } \quad b_{k}=\left(\mathbf{x}, \mathbf{h}_{k}\right) /\left(\mathbf{h}_{k}, \mathbf{h}_{k}\right)
$$

To each decomposition (4.5) with $0 \leq j \leq n$, there corresponds a partition of unity $\left\{\mathbf{n}_{j, k} \in L_{N}\left(I^{d}\right): 0 \leq k<2^{j}\right\}$, where

$$
\begin{gathered}
n_{j, k}(i)= \begin{cases}1 & \text { for } i \in I_{j, k}, \\
0 & \text { for } i \in \mathbb{Z} \backslash I_{j, k},\end{cases} \\
n_{j, k}(i)=n_{0,0}\left(2^{j} i-k 2^{n}\right), \\
\sum_{k=0}^{2^{j}-1} \mathbf{n}_{j, k}=\mathbf{1} \quad \text { on } I .
\end{gathered}
$$

Note that (4.6) implies

$$
\mathbf{n}_{n, k}=\mathbf{e}_{k} \quad \text { for } k \in I,
$$

and for $0 \leq j<n,(4.7)$ gives

$$
\mathbf{n}_{j, k}=\mathbf{n}_{j+1,2 k}+\mathbf{n}_{j+1,2 k+1} \quad \text { for } 0 \leq k<2^{j} \text {. }
$$


Let us introduce in $L_{n}(I)$ an increasing sequence of subspaces by setting

$$
L_{j}(I)=\operatorname{span}\left\{\mathbf{n}_{j, k}: 0 \leq k<2^{j}\right\}, \quad 0 \leq j \leq n .
$$

The inclusion $L_{j}(I) \subset L_{j+1}(I)$ follows by (4.17). Clearly, the dimension of $L_{j}(I)$ is $2^{j}$. There are important relations between the Haar orthogonal basis and the family of partitions of unity: for $0 \leq s<2^{j}$ and $0 \leq j \leq n$,

$$
\left|\mathbf{h}_{2^{j}+s}\right|=\mathbf{n}_{j, s}=\mathbf{n}_{j+1,2 s}+\mathbf{n}_{j+1,2 s+1}
$$

where $\left|\mathbf{h}_{m}\right|=\left\{\left|h_{m}(i)\right|: i \in I\right\}$, and for $2^{j} \leq t<2^{j+1}$ and $u=t-2^{j}$,

$$
\mathbf{h}_{t}=\mathbf{h}_{2^{j}+u}=\mathbf{n}_{j+1,2 u}-\mathbf{n}_{j+1,2 u+1} .
$$

To define the Haar transform some more notation is needed. It is convenient to consider for $\underline{i} \in I^{d}$ the function $\vee \underline{i}=\max \left(i_{1}, \ldots, i_{d}\right)$. We now decompose $I^{d}$ as follows:

where

$$
I^{d}=\{\underline{0}\} \cup \bigcup_{j=0}^{n} J_{j},
$$

Each of the sets in (4.22) is further decomposed as

$$
J_{j}=\bigcup_{\emptyset \neq e \subset D} J_{j, e},
$$

where

$$
J_{j, e}=\left\{\underline{i} \in I^{d}: 2^{j} \leq i_{k}<2^{j+1} \text { if } k \in e \text { and } i_{k}<2^{j} \text { if } k \in D \backslash e\right\} .
$$

Now, define the tensor product $\mathbf{x}_{1} \otimes \ldots \otimes \mathbf{x}_{d}$ of $\mathbf{x}_{1}, \ldots, \mathbf{x}_{d} \in L_{n}(I)$ as an element of $L_{n}\left(I^{d}\right)$ such that

$$
\left(x_{1} \otimes \ldots \otimes x_{d}\right)(\underline{i})=x_{1}\left(i_{1}\right) \ldots x_{d}\left(i_{d}\right) \quad \text { for } \underline{i} \in I^{d} .
$$

Definition 4.2. The discrete d-dimensional Haar system is defined as follows:

$$
\mathbf{h}_{\underline{0}}=\bigotimes_{k=1}^{d} \mathbf{h}_{0}
$$

and for $\underline{i} \in J_{j, e}$,

$$
\mathbf{h}_{\underline{i}}=\bigotimes_{k \in e} \mathbf{h}_{i_{k}} \otimes \bigotimes_{k \in D \backslash e}\left|\mathbf{h}_{2^{j}+i_{k}}\right| .
$$

As in the one-dimensional case, for any $\mathbf{u}, \mathbf{v} \in L_{n}\left(I^{d}\right)$ we have the scalar product

$$
(\mathbf{u}, \mathbf{v})=\frac{1}{\left|I^{d}\right|} \sum_{\underline{i} \in I^{d}} u(\underline{i}) v(\underline{i}) .
$$


In analogy to (4.18) we introduce an increasing sequence of subspaces in $L_{n}\left(I^{d}\right)$ by defining, for $0 \leq j \leq n$,

$$
L_{j}\left(I^{d}\right)=\operatorname{span}\left\{\mathbf{n}_{j, \underline{i}}: \underline{i} \in I^{d} \text { and } \vee \underline{i}<2^{j}\right\},
$$

where

$$
\mathbf{n}_{j, \underline{i}}=\bigotimes_{k=1}^{d} \mathbf{n}_{j, i_{k}}
$$

Proposition 4.1. The d-dimensional Haar system $\left\{\mathbf{h}_{\underline{i}}: \underline{i} \in I^{d}\right\}$ has the following properties: it is orthogonal, $\left(\mathbf{h}_{\underline{i}}, \mathbf{h}_{\underline{i}}\right)=2^{-j d}$ for $\underline{i} \in J_{j}$ and

$$
L_{j}\left(I^{d}\right)=\operatorname{span}\left\{\mathbf{h}_{\underline{i}}: \underline{i} \in\{\underline{0}\} \cup \bigcup_{s=1}^{j-1} J_{s}\right\} .
$$

Moreover, for the support of $\mathbf{h}_{\underline{i}}$ we have the formula

$$
I_{\underline{i}}=\prod_{\nu=1}^{d}\left[2^{n-j} k_{\nu}, 2^{n-j}\left(k_{\nu}+1\right)\right) \quad \text { with } \quad k_{\nu}=i_{\nu} \bmod 2^{j}
$$

There are always $2^{d}-1$ Haar functions having the same support; $\mathbf{h}_{\underline{0}}$ is an exception.

Each $\mathbf{y} \in L_{n}\left(I^{d}\right)$ can be represented in the Haar basis $\left\{\mathbf{h}_{\underline{i}}: \underline{i} \in I^{d}\right\}$ as

$$
\mathbf{y}=\sum_{\underline{i} \in I^{d}} b(\underline{i}) \mathbf{h}_{\underline{i}}=b(\underline{0}) \mathbf{h}_{\underline{0}}+\sum_{j=0}^{n} \sum_{\underline{i} \in J_{j}} b(\underline{i}) \mathbf{h}_{\underline{i}},
$$

where

$$
\begin{aligned}
b(\underline{0}) & =\left(\mathbf{y}, \mathbf{h}_{\underline{0}}\right), \\
b(\underline{i}) & =\left(2^{j}\right)^{d}\left(\mathbf{y}, \mathbf{h}_{\underline{i}}\right) \quad \text { for } \underline{i} \in J_{j}, 0 \leq j \leq n .
\end{aligned}
$$

Definition 4.3. The map $\mathbf{H}: \mathbf{y} \mapsto \mathbf{b}$ given by (4.33) is called the Haar transform.

Our next goal is to extract from formulas (4.34) and (4.35) an algorithm for calculating $\mathbf{b}$ in terms of $\mathbf{y}$.

Proposition 4.2. To calculate $\mathbf{b}=\mathbf{H y}$ we have the following formulas:

$$
b(\underline{0})=\frac{1}{\left|I^{d}\right|} \sum_{\underline{i} \in I^{d}} y(\underline{i})
$$

and if $\underline{i} \in J_{j, e}$, then

$$
b(\underline{i})=\frac{1}{\left|I_{\underline{i}}\right|} \sum_{\underline{\eta}}(-1)^{|\underline{\eta}|} \sum_{\underline{m}^{\prime \prime} \in I_{\underline{\eta}}} y_{e}\left(\underline{m}^{\prime \prime}\right),
$$


where $I_{\underline{i}}$ is given as in (4.32), $\eta$ runs over 0,1 sequences of length $|e|, y_{e}$ is defined as in (4.41) and $I_{\underline{\eta}}$ as in (4.42). Moreover,

$$
|b(\underline{i})| \leq \max \left\{\left|y\left(\underline{i}^{\prime}\right)\right|: \underline{i}^{\prime} \in I^{d}\right\} \quad \text { for } \underline{i} \in I^{d} .
$$

Proof. Formula (4.35) for $\underline{i} \in J_{j}$ can be rewritten as

$$
b(\underline{i})=\frac{1}{\left|I_{\underline{i}}\right|} \sum_{\underline{m} \in I_{\underline{i}}} y(\underline{m}) h_{\underline{i}}(\underline{m}) .
$$

Now, by (4.6) and (4.7) for $\underline{i} \in J_{j, e}$ obtain

$$
I_{\underline{i}}=\prod_{\nu \in D \backslash e} I_{j, k_{\nu}} \times \bigcup_{\underline{\eta}} \prod_{\nu \in e} I_{j+1,2 k_{\nu}+\eta_{\nu}}
$$

where $\underline{\eta}=\left(\eta_{1}, \ldots, \eta_{|e|}\right)$ with $\eta_{\nu}=0,1$. Consequently, $I_{\underline{i}}=I^{\prime} \times I^{\prime \prime}$, and so $\underline{m} \in I_{\underline{i}}$ if and only if $\underline{m}=\left(\underline{m}^{\prime}, \underline{m}^{\prime \prime}\right)$ with $\underline{m}^{\prime} \in I^{\prime}$ and $\underline{m}^{\prime \prime} \in I^{\prime \prime}$ where

Define now

$$
I^{\prime}=\prod_{\nu \in D \backslash e} I_{j, k_{\nu}}, \quad I^{\prime \prime}=\bigcup_{\underline{\eta}} \prod_{\nu \in e} I_{j+1,2 k_{\nu}+\eta_{\nu}} .
$$

$$
y_{e}\left(\underline{m}^{\prime \prime}\right)=\sum_{\underline{m}^{\prime} \in I^{\prime}} y\left(\underline{m}^{\prime}, \underline{m}^{\prime \prime}\right)
$$

Clearly,

$$
\sum_{\underline{m} \in I_{\underline{i}}} y(\underline{m}) h_{\underline{i}}(\underline{m})=\sum_{\underline{\eta}} \sum_{\underline{m}^{\prime \prime} \in I_{\underline{\eta}}} y_{e}\left(\underline{m}^{\prime \prime}\right) \prod_{\nu \in e} h_{2^{j}+k_{\nu}}\left(m_{\nu}\right),
$$

where

$$
I_{\underline{\eta}}=\prod_{\nu \in e} I_{j+1,2 k_{\nu}+\eta_{\nu}} .
$$

Thus,

$$
\sum_{\underline{m} \in I_{\underline{i}}} y(\underline{m}) h_{\underline{i}}(\underline{m})=\sum_{\underline{\eta}}(-1)^{|\underline{\mid \eta}|} \sum_{\underline{m}^{\prime \prime} \in I_{\underline{\eta}}} y_{e}\left(\underline{m}^{\prime \prime}\right) ;
$$

comparing this with (4.39) we find (4.37) and the proof is complete.

In the two-dimensional case formula (4.37) becomes very simple.

Our next goal is to present an algorithm for calculating the map $\mathbf{G}=$ $\mathbf{H}^{-1}$, inverse to the Haar transform. We apply the so-called bottom up algorithm. Using the notation introduced earlier we assume that we are given $\mathbf{b} \in L_{n}\left(I^{d}\right)$ and we want to find $\mathbf{y}=\mathbf{G b}$ given by (4.33). To construct the algorithm we introduce the partial sums for $\mathbf{y}$ :

$$
\begin{aligned}
& \mathbf{y}^{(0)}=b(\underline{0}) \mathbf{h}_{\underline{0}}, \\
& \mathbf{y}^{(s)}=\mathbf{y}^{(0)}+\sum_{j=0}^{s-1} \sum_{\underline{i} \in J_{j}} b(\underline{i}) \mathbf{h}_{\underline{i}} \quad \text { for } s=1, \ldots, n .
\end{aligned}
$$


Clearly,

$$
\mathbf{y}^{(n)}=\mathbf{y}, \quad \mathbf{y}^{(s)} \in L_{s}\left(I^{d}\right) \quad \text { for } s=0, \ldots, n .
$$

It follows from (4.45) and (4.29) that $\mathbf{y}^{(s)}$ can be represented in the basis $\left\{\mathbf{n}_{s, \underline{i}}: \underline{i} \in I^{d}\right.$ and $\left.\vee \underline{i}<2^{s}\right\}$ :

$$
\mathbf{y}^{(s)}=\sum_{\vee \underline{i}<2^{s}} b^{(s)}(\underline{i}) \mathbf{n}_{s, \underline{i}} .
$$

Here and later on we denote by $\vee \underline{i}<2^{s}$ the set $\left\{\underline{i} \in I^{d}: \vee \underline{i}<2^{s}\right\}$. We are going to determine the $b^{(s)}(\underline{i})$ recursively in terms of the Haar coefficients $b(\underline{i})$ in (4.33). For $s=0$ we have

$$
b^{(0)}(\underline{0})=b(\underline{0}) .
$$

Now, (4.44) gives

$$
\mathbf{y}^{(s+1)}=\mathbf{y}^{(s)}+\sum_{\underline{i} \in J_{s}} b(\underline{i}) \mathbf{h}_{\underline{i}} \quad \text { for } s=0, \ldots, n .
$$

Using (4.17), for $\underline{i} \in I^{d}$ we get

$$
\mathbf{n}_{s, \underline{i}}=\sum_{\underline{\varepsilon}} \mathbf{n}_{s+1,2 \underline{i}+\underline{\varepsilon}},
$$

where $\underline{\varepsilon}=\left(\varepsilon_{1}, \ldots, \varepsilon_{d}\right)$ with $\varepsilon_{\nu}=0,1$. Thus (4.46) can be rewritten as

$$
\mathbf{y}^{(s)}=\sum_{\vee \underline{i}<2^{s}} b^{(s)}(\underline{i}) \sum_{\underline{\varepsilon}} \mathbf{n}_{s+1,2 \underline{i}+\underline{\varepsilon}} .
$$

Introducing the indicator function $\chi_{\underline{i}}(\underline{m})$ of the set

$$
\left\{2 i_{1}, 2 i_{1}+1\right\} \times \ldots \times\left\{2 i_{d}, 2 i_{d}+1\right\},
$$

and changing the order of summation in (4.50), we get

$$
\mathbf{y}^{(s)}=\sum_{\vee \underline{m}<2^{s+1}} \sum_{\vee \underline{i}<2^{s}} b^{(s)}(\underline{i}) \chi_{\underline{i}}(\underline{m}) \mathbf{n}_{s+1, \underline{m}} .
$$

Now, the equality $\chi_{\underline{i}}(\underline{m})=1$ implies $\underline{i}=\lfloor\underline{m} / 2\rfloor$; here for $\underline{c}=\left(c_{1}, \ldots, c_{d}\right)$ we define $\lfloor\underline{c}\rfloor=\left(\left\lfloor\underline{c}_{1}\right\rfloor, \ldots,\left\lfloor\underline{c}_{d}\right\rfloor\right)$. Thus, (4.51) can be simplified to

$$
\mathbf{y}^{(s)}=\sum_{\vee \underline{m}<2^{s+1}} b^{(s)}(\lfloor\underline{m} / 2\rfloor) \mathbf{n}_{s+1, \underline{m}} .
$$

It also follows by (4.17), (4.19), (4.20) and (4.27) that each $\mathbf{h}_{\underline{i}}, \underline{i} \in J_{s}$, has an explicit representation in the basis $\left\{\mathbf{n}_{s+1, \underline{m}}: \vee \underline{m}<2^{s+1}\right\}$. Indeed, defining $\underline{k}=\underline{i} \bmod 2^{s}$ we find that 


$$
\begin{aligned}
\mathbf{h}_{\underline{i}} & =\bigotimes_{\nu=1}^{d}\left(n_{s+1,2 k_{\nu}}+(-1)^{\varepsilon_{s}\left(i_{\nu}\right)} n_{s+1,2 k_{\nu}+1}\right) \\
& =\sum_{\underline{\eta}} \bigotimes_{\nu=1}^{d}(-1)^{\eta_{\nu} \varepsilon_{s}\left(i_{\nu}\right)} n_{s+1,2 k_{\nu}+\eta_{\nu}} \\
& =\sum_{\underline{\eta}}(-1)^{\eta_{1} \varepsilon_{s}\left(i_{1}\right)+\ldots+\eta_{d} \varepsilon_{s}\left(i_{d}\right)} \mathbf{n}_{s+1,2 \underline{k}+\underline{\eta}} \\
& =\sum_{\underline{\eta}}(-1)^{(2 \underline{k}+\underline{\eta}) \cdot \underline{\varepsilon}_{s}} \mathbf{n}_{s+1,2 \underline{k}+\underline{\eta}} \\
& =\sum_{\vee \underline{m}<2^{s+1}}(-1)^{\underline{m} \cdot \underline{\varepsilon}_{s}} \chi_{\underline{k}}(\underline{m}) \mathbf{n}_{s+1, \underline{m}},
\end{aligned}
$$

where $\varepsilon_{s}(i)$ is given by the dyadic expansion $i=\varepsilon_{0}(i)+\varepsilon_{1}(i) \cdot 2+\varepsilon_{2}(i) \cdot 2^{2}+\ldots$; $(2 \underline{k}+\underline{\eta}) \cdot \underline{\varepsilon}_{s}$ is the dot product of $2 \underline{k}+\underline{\eta}$ and $\underline{\varepsilon}_{s}=\left(\varepsilon_{s}\left(i_{1}\right), \ldots, \varepsilon_{s}\left(i_{d}\right)\right)$; $\underline{\eta}=\left(\eta_{1}, \ldots, \eta_{d}\right)$ with $\eta_{\nu}=0,1$; and $\chi_{\underline{k}}(\underline{m})$ is the indicator function of the product set $\left\{\underline{m}: 2 k_{\nu} \leq m_{\nu} \leq 2 k_{\nu}+1\right.$ for $\left.\nu=1, \ldots, d\right\}$. Consequently,

$$
\sum_{\underline{i} \in J_{s}} b(\underline{i}) \mathbf{h}_{\underline{i}}=\sum_{\vee \underline{m}<2^{s+1}} \sum_{\underline{i} \in J_{s}} b(\underline{i})(-1)^{\underline{m} \cdot \underline{\varepsilon}_{s}} \chi_{\underline{k}}(\underline{m}) \mathbf{n}_{s+1, \underline{m}} .
$$

Now, for fixed $\underline{m}$ the equality $\chi_{\underline{k}}(\underline{m})=1$ implies that $\underline{m}=2 \underline{k}+\eta$ and that $\underline{k}=\lfloor\underline{m} / 2\rfloor$ and $\underline{i}=\lfloor\underline{m} / 2\rfloor+\underline{\varepsilon}_{s} 2^{s}$ with $\left|\underline{\varepsilon}_{s}\right|=\left|\varepsilon_{s}\left(i_{1}\right)\right|+\ldots+\left|\overline{\varepsilon_{s}}\left(i_{d}\right)\right|>0$. Thus,

where

$$
\sum_{\underline{i} \in J_{s}} b(\underline{i}) \mathbf{h}_{\underline{i}}=\sum_{\vee \underline{m}<2^{s+1}} B^{(s+1)}(\underline{m}) \mathbf{n}_{s+1, \underline{m}}
$$

$$
B^{(s+1)}(\underline{m})=\sum_{\underline{\eta}:|\underline{\eta}|>0}(-1)^{\underline{\eta}} \cdot \underline{m} b\left(\lfloor\underline{m} / 2\rfloor+\underline{\eta} \cdot 2^{s}\right) .
$$

where $\underline{\eta}$ runs through all the 0,1 sequences of length $d$. Using now (4.46), (4.48), (4.52), (4.54) and (4.55) we obtain the basic recurrence relation

$$
b^{(s+1)}(\underline{m})=b^{(s)}(\lfloor\underline{m} / 2\rfloor)+B^{(s+1)}(\underline{m})
$$

for $\vee \underline{m}<2^{s+1}$ and $s=0, \ldots, n$. In particular, since $\mathbf{y}=\mathbf{y}^{(n+1)}$, we obtain the value of the inverse Haar transform Gb.

In the final step of our discussion we treat the Haar compression, introducing, motivated by the theory, the corresponding Besov norm

$$
\|\mathbf{y}\|_{p}^{(\alpha)}=\left(|b(\underline{0})|^{p}+\sum_{j=0}^{n} 2^{j(\alpha p-d)} \sum_{\underline{i} \in J_{j}}|b(\underline{i})|^{p}\right)^{1 / p},
$$

where the coefficients $\{b(\underline{i})\}$ are given by (4.33). It now follows from (4.57) that 


$$
\left\|\mathbf{h}_{\underline{\underline{i}}}\right\|_{p}^{(\alpha)}=2^{j(\alpha-d / p)} \quad \text { for } \underline{i} \in J_{j} .
$$

Let us introduce the Haar system $\mathbf{h}_{\underline{0}}^{(\alpha, p)}=\mathbf{1}, \mathbf{h}_{\underline{i}}^{(\alpha, p)}=2^{j(d / p-\alpha)} \mathbf{h}_{\underline{i}}$ whenever $\underline{i} \in J_{j}$ and $j=0, \ldots, n$. It is the Haar basis normalized in the Besov norm $\|\cdot\|_{p}^{(\alpha)}$ and the representation (4.33) now takes the form

$$
\mathbf{y}=b^{(\alpha, p)}(\underline{0}) \mathbf{h}_{\underline{0}}^{(\alpha, p)}+\sum_{j=0}^{n} \sum_{\underline{i} \in J_{j}} b^{(\alpha, p)}(\underline{i}) \mathbf{h}_{\underline{i}}^{(\alpha, p)}=\sum_{\vee \underline{i}<2^{n}} b^{(\alpha, p)}(\underline{i}) \mathbf{h}_{\underline{i}}^{(\alpha, p)},
$$

where

$$
b^{(\alpha, p)}(\underline{0})=b(\underline{0}), \quad b^{(\alpha, p)}(\underline{i})=2^{j(\alpha-d / p)} b(\underline{i}), \quad \underline{i} \in J_{j}, 0 \leq j \leq n .
$$

The space $L_{n}\left(I^{d}\right)$ with the Besov norm $\|\cdot\|_{p}^{(\alpha)}$ becomes $l_{2^{n d}}^{p}$ and (4.57) reads

$$
\begin{aligned}
\|\mathbf{y}\|_{p}^{(\alpha)} & =\left[\left|b^{(\alpha, p)}(\underline{0})\right|^{p}+\sum_{j=0}^{n} \sum_{\underline{i} \in J_{j}}\left|b^{(\alpha, p)}(\underline{i})\right|^{p}\right]^{1 / p} \\
& =\left[\sum_{\vee \underline{i}<2^{n}}\left|b^{(\alpha, p)}(\underline{i})\right|^{p}\right]^{1 / p} .
\end{aligned}
$$

We recall that we have a good motivation for these considerations for the parameters $(\alpha, p)$ in the range $0<\alpha<1 / p \leq 1$.

To conclude our compression we introduce

$$
B(\lambda)=\left\{\bigvee \underline{i}<2^{n}:\left|b^{(\alpha, p)}(\underline{i})\right| \leq \lambda\right\}, \quad \lambda \geq 0,
$$

and the distribution function

$$
F(\lambda)=|B(\lambda)|
$$

Now, as in the diamond case we have

Proposition 4.3. Assume that $0<\alpha<1 / p \leq 1$. For given $\alpha, p$ and $\lambda \geq 0$ we assign to $\mathbf{y}$ given by (4.59) the compressed $\mathbf{y}$ :

$$
\mathbf{y}_{\lambda}=\sum_{\vee \underline{i}<2^{n}} b_{\lambda}^{(\alpha, p)}(\underline{i}) \mathbf{h}_{\underline{i}}^{(\alpha, p)},
$$

where

$$
b_{\lambda}^{(\alpha, p)}(\underline{i})= \begin{cases}b^{(\alpha, p)}(\underline{i}) & \text { for } \underline{i} \in I^{d} \backslash B(\lambda) \\ 0 & \text { for } B(\lambda)\end{cases}
$$

Then

$$
\left\|\mathbf{y}-\mathbf{y}_{\lambda}\right\|_{p}^{(\alpha)} \leq \lambda[F(\lambda)]^{1 / p},
$$

and equality in (4.66) is attained in particular for $\mathbf{y}$ of the form

$$
\mathbf{y}_{\lambda}=\sum_{\vee \underline{i}<2^{n}} \pm \lambda \mathbf{h}_{\underline{i}}^{(\alpha, p)} .
$$


The definition of $\varepsilon$-compression is the same as in Definition 3.4. Moreover, as in the diamond case we have the same

Corollary 4.4. If $\lambda[F(\lambda)]^{1 / p} \leq \varepsilon$, then $\mathbf{y}_{\lambda}$ is an $\varepsilon$-compression.

\section{References}

[1] Z. Ciesielski, Haar orthogonal functions in analysis and probability, in: A. Haar Memorial Conference (Budapest, 1985), Colloq. Math. Soc. János Bolyai 49, NorthHolland, 1987, 25-56.

[2] Z. Ciesielski and A. Kamont, Lévy's fractional Brownian random field and function spaces, Acta Sci. Math. (Szeged) 60 (1995), 99-118.

[3] R. A. DeVore, B. Jawerth and B. Lucier, Image compression through wavelet transform coding, IEEE Trans. Inform. Theory 38 (1992), 719-746.

[4] S. V. Konyagin and V. N. Temlyakov, A remark on greedy approximation in Banach spaces, East J. Approx. 5 (1999), 365-379.

[5] J. Ryll, Schauder bases for the space of continuous functions on an n-dimensional cube, Comment. Math. Prace Mat. 27 (1973), 201-213.

[6] J. Schauder, Eine Eigenschaft des Haarschen Orthogonalsystems, Math. Z. 28 (1928), $317-320$.

[7] Z. Semadeni, Schauder Bases in Banach Spaces of Continuous Functions, Lecture Notes in Math. 918, Springer, Berlin, 1982.

[8] I. Singer, Bases in Banach Spaces I, Springer, Berlin, 1982.

B. Ciesielski

E-mail: bolek@ne.mediaone.net
Z. Ciesielski

Institute of Mathematics Polish Academy of Sciences

Abrahama 18

81-825 Sopot, Poland

E-mail: Z.Ciesielski@impan.gda.pl

Received on 21.6.2001;

revised version on 12.9.2001 